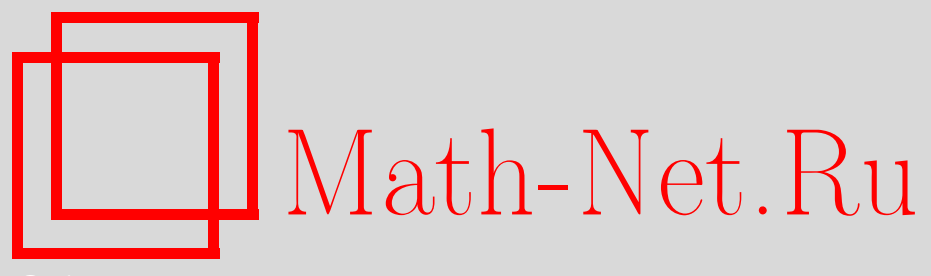

В. А. Артамонов, В. М. Бухштабер, Э. Б. Винберг, Л. В. Кузьмин, В. С. Куликов, В. Н. Латышев, А. В. Михалев, А. Ю. Ольшанский, Д. О. Орлов, А. Н. Паршин, Д. И. Пионтковский, Евгений Соломонович Голод (некролог), УМH, 2019, том 74, выпуск 5, 163-169

DOI: https://doi.org/10.4213/rm9878

Использование Общероссийского математического портала Math-Net.Ru подразумевает, что вы прочитали и согласны с пользовательским соглашением http://www . mathnet.ru/rus/agreement

Параметры загрузки:

IP : 52.6 .47 .48

26 апреля 2023 г., 14:24:06 


\section{Евгений Соломонович Голод}

5 июля 2018 г. после непродолжительной тяжелой болезни на 83-м году жизни скончался известный советский и российский алгебраист Евгений Соломонович Голод.

Он родился 21 октября 1935 г. в Москве в семье служащих. В 1953 г. Е.С. Голод окончил с золотой медалью школу в г. Иваново и поступил на механико-математический факультет МГУ, где на первом курсе начал посещать семинар И.Р. Шафаревича и вскоре стал его учеником.

После окончания мехмата Е. С. Голод поступил в аспирантуру того же факультета, по окончании которой в 1964 г. защитил кандидатскую диссертацию "О гомологиях конечных $p$-групп и локальных колец". Затем в течение ряда лет Е.С. Голод преподавал в Текстиль-

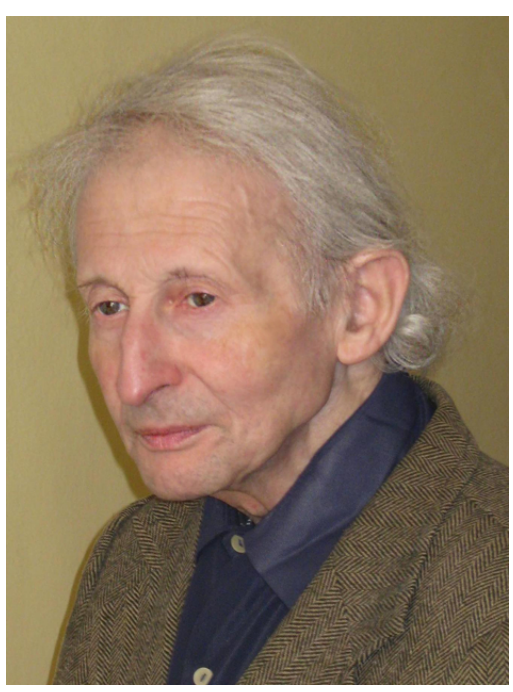
ном институте в Москве и его филиале в подмосковном городе Павловский Посад.

В 1961 г. он по приглашению А. Г. Куроша начал работать на кафедре высшей алгебры механико-математического факультета МГУ сначала в должности ассистента, а с 1966 г. в должности доцента.

В 1999 г. Евгений Соломонович защитил докторскую диссертацию под названием “Комплекс Шафаревича и его приложения"; с 2000 г. он работал на кафедре высшей алгебры в должности профессора, получив в 2005 г. звание профессора, а в 2011 г. звание заслуженного профессора Московского университета. Его лаконичный точный стиль изложения научного и учебного материала был отточен десятилетиями работы в реферативном журнале "Математика", где Евгений Соломонович был редактором одного из разделов. Кроме того, эта работа в реферативном журнале была неоценимо полезна с точки зрения получения советскими (а с 1990-х годов российскими) математиками своевременной информации о новых результатах зарубежных ученых.

Научные интересы Евгения Соломоновича были сосредоточены вокруг гомологической и коммутативной алгебры. Всего им опубликованы 23 научные работы. Несмотря на небольшой общий объем, эти работы содержат целый ряд красивых и ярких результатов, многие из которых активно используются и развиваются и по сей день.

DOI: https://doi.org/10.4213/rm9878 
Свою первую научную работу [1] Евгений Соломонович написал, будучи студентом третьего курса мехмата МГУ. В этой работе дан положительный ответ на вопрос, заданный в книге Картана-Эйленберга ${ }^{1}$, о существовании нётеровых проективных, но несвободных модулей над целочисленным групповым кольцом $\mathbb{Z}[G]$ конечной группы $G$. Пусть $G$ - циклическая группа порядка $p$, где $p$ - такое простое число, что поле деления круга на $p$ частей многоклассно. В этом случае строятся два несвободных $\mathbb{Z}[G]$-модуля $M_{1}$ и $M_{2}$ такие, что

$$
M_{1} \oplus M_{2} \cong \mathbb{Z}[G] \oplus \mathbb{Z}[G]
$$

т. е. $M_{1}$ и $M_{2}$ проективны.

Затем последовали новые яркие теоремы: доказательство конечной порожденности алгебры когомологий конечной $p$-группы [2] (эта теорема была вскоре обобщена Б. А. Венковым и Л. Ивенсом на произвольные конечные группы) и описание математических объектов, которые с тех пор носят название "колец Голода" [3] - локальных и градуированных колец с максимальными числами Бетти при данных размерностях гомологий комплекса Козюля. Если $A$ - коммутативное нётерово локальное кольцо и минимальная система образующих его максимального идеала $\mathfrak{m}$ состоит из $m$ элементов, то для функции Пуанкаре $F_{A}(t)$ кольца $A$ выполняется неравенство

$$
F_{A}(t) \leqslant \frac{(1+t)^{m}}{1-\sum c_{i} t^{i+1}}
$$

где $c_{i}$ - размерность группы гомологий $H_{i}(K)$ комплекса Козюля $K$. Это неравенство было открыто Ж.-П. Серром, и, таким образом, Е. С. Голод ответил на вопрос, когда это неравенство является точным. Доказано, что это неравенство превращается в равенство тогда и только тогда, когда алгебра гомологий $H(K)$ комплекса Козюля имеет нулевое умножение и все операции Масси, соответствующие симплексам, равны нулю.

Доказательство основано на том, что указанное выше неравенство становится равенством тогда и только тогда, когда числа Бетти кольца $A$ удовлетворяют некоторому рекуррентному соотношению, которое можно также выразить в терминах минимальной проективной резольвенты $A$-модуля $k=A / \mathfrak{m}$. Указанная резольвента строится в явном виде, причем оказывается, что построение выполнимо тогда и только тогда, когда все умножения в $H(K)$ нулевые, равно как и все операции Масси, соответствующие симплексам, т. е. (в современной терминологии) структура $A_{\infty}$-алгебры на гомологиях тривиальна. Эти результаты составили основу его кандидатской диссертации. Они стали широко известными и легли в основу понятий "кольца Голода" в гомологической алгебре и "симплициальные комплексы Голода" в торической топологии.

Следующая работа [4] Евгения Соломоновича, выполненная совместно с И. Р. Шафаревичем, можно без преувеличения сказать, принесла Е. С. Голоду мировую известность. Эта работа содержит отрицательное решение знаменитой проблемы о башне полей классов. Еще Гильберт предположил (и доказал в частных случаях), что для любого поля алгебраических чисел $K$ его максимальное абелево неразветвленное расширение $K_{1}$ имеет конечную степень над $K$ и группа Галуа $G\left(K_{1} / K\right)$ изоморфна группе классов идеалов поля $K$. Более того, все идеалы поля $K$ становятся главными

${ }^{1}$ См. А. Картан, С. Эйленберг, Гомологическая алгебра, ИЛ, М. 1960, 510 с.; пер. с англ.: H. Cartan, S. Eilenberg, Homological algebra, Princeton Univ. Press, Princeton, NY, 1956, $\mathrm{xv}+390 \mathrm{pp}$. 
в $K_{1}$. Позже эти гипотезы были доказаны, а поле $K_{1}$ получило название гильбертова поля классов поля $K$.

Для поля $K_{1}$ можно рассмотреть его гильбертово поле классов $K_{2}$ и т. д. Таким образом получаем цепочку полей

$$
K \subseteq K_{1} \subseteq K_{2} \subseteq \cdots,
$$

называемую башней полей классов. Проблема башни полей классов заключалась в вопросе о том, всегда ли эта башня конечна. Это эквивалентно утверждению, что любое поле алгебраических чисел $K$ можно вложить в одноклассное поле, и простейшие примеры как будто подсказывали, что так оно и есть.

Новый подход к этой проблеме был предложен в работе И. Р. Шафаревича "Расширения с заданными точками ветвления" 2 . В этой работе для некоторого фиксированного простого $p$ рассматривалась так называемая $p$-башня полей классов, т. е. вместо гильбертова поля классов $K_{1}$ поля $K$ рассматривалось $p$-гильбертово поле классов $K_{1}^{(p)}$ - максимальное подполе в $K_{1}$ такое, что $K_{1}^{(p)} / K$ является $p$-расширением. Если $K_{2}^{(p)}-p$-гильбертово поле классов поля $K_{1}^{(p)}$ и, вообще, $K_{i+1}^{(p)}-p$-гильбертово поле классов поля $K_{i}^{(p)}$, то цепочка полей

$$
K \subseteq K_{1}^{(p)} \subseteq \cdots \subseteq K_{i}^{(p)} \subseteq \cdots
$$

называется $p$-башней полей классов. Для отрицательного решения проблемы башни полей классов достаточно доказать бесконечность $p$-башни полей классов для некоторого простого $p$. Пусть $K_{\infty}^{(p)}=\bigcup_{i=1}^{\infty} K_{i}^{(p)}$ - максимальное неразветвленное $p$-расширение поля $K$ и $G$ - группа Галуа расширения $K_{\infty}^{(p)} / K$. Тогда $G$ является про-р-группой. Используя теорию полей классов, И.Р. Шафаревич получил в указанной работе для $G$ как про-р-группы оценку минимального числа образующих $d=d(G)$ и минимального числа соотношений $r=r(G)$. В частности, он показал, что всегда выполняется неравенство $r-d \leqslant \rho+\delta$, где $\rho$ - ранг группы единиц поля $K$, а $\delta=0$, если $\zeta_{p} \notin K$, и $\delta=1$ в противном случае. Здесь $\zeta_{p}-$ первообразный корень из единицы степени $p$. Таким образом, если, например, $K$ - циклическое расширение поля $\mathbb{Q}$ степени $p$, то $d$ равно минимальному числу образующих $p$-группы классов поля $K$, и $d$ может быть сколь угодно велико, если дискриминант $K$ содержит достаточно много различных простых делителей, а $r-d \leqslant p-1$. Это следует из теоремы Дирихле о единицах (если $K$ - вещественное квадратичное поле, то $r-d \leqslant 2$ ).

Рассматривая примеры различных конечных $p$-групп, И. Р. Шафаревич предположил, что для любой бесконечной последовательности конечных $p$-групп $\left\{G_{i}\right\}$ такой, что $\lim _{i \rightarrow \infty} d\left(G_{i}\right)=\infty$, должно также выполняться и условие

$$
\lim _{i \rightarrow \infty}\left(r\left(G_{i}\right)-d\left(G_{i}\right)\right)=\infty
$$

По существу, доказательству этой гипотезы и была посвящена работа [4]. В ней было доказано знаменитое неравенство Голода-Шафаревича

$$
r(G)>\left(\frac{d(G)-1}{2}\right)^{2}
$$

которое справедливо для любой конечной $p$-группы $G$.

\footnotetext{
${ }^{2}$ Publ. Math. Inst. Hautes Études Sci., 18 (1963), 295-319.
} 
Доказательство основано на переформулировке задачи в терминах групповой алгебры $A$ группы $G$ над полем из $p$ элементов, которая является факторалгеброй алгебры некоммутативных многочленов от $d$ переменных. Рассматриваются когомологии некоторого комплекса $M$, связанного с $A$, который является некоммутативным аналогом комплекса Козюля и называется сейчас комплексом Шафаревича.

Е. С. Голод получает неравенства, связывающие коэффициенты некоторых рядов Пуанкаре, определяемых в терминах когомологий комплекса $M$, и доказывает, что эти неравенства не могут выполняться для конечномерной алгебры $A$, если не выполняется неравенство $(*)$.

В [4] приводится простейший пример поля, имеющего бесконечную $p$-башню полей классов (для $p=2$ ). Таковым является мнимое квадратичное поле

$$
k=\mathbb{Q}(\sqrt{-3 \cdot 5 \cdot 7 \cdot 11 \cdot 13 \cdot 17 \cdot 19}) .
$$

Интересно отметить, что даже сейчас, спустя более полувека, теорема Голода-Шафаревича остается самым глубоким результатом, относящимся к неразветвленным расширениям полей алгебраических чисел.

В работе [5] Е.С. Голод, используя идеи и результаты работы [4], строит пример ассоциативной ненильпотентной ниль-алгебры с конечным числом образующих и пример бесконечной финитно-аппроксимируемой $p$-группы с конечным числом образующих. Первый из этих примеров дает отрицательный ответ на вопрос А. Г. Куроша о том, будет ли локально конечномерной ассоциативная алгебраическая алгебра, т. е. ассоциативная алгебра, в которой каждый элемент алгебраичен, а также на вопрос Я. Левицкого о локальной нильпотентности ассоциативного нилькольца. Второй пример дает отрицательное решение общей проблемы Бернсайда о периодических группах, а именно, существуют бесконечные конечно порожденные (и даже финитно-аппроксимируемые) $p$-группы.

Полученное Е. С. Голодом решение целого ряда известных проблем бернсайдовского типа стало в середине 1960-х годов сенсацией и сделало понятие группы Голода известным каждому алгебраисту. Обобщение и развитие этой конструкции до сих пор приносит решение все новых проблем в теории колец и в теории групп.

Методу построения подобных контрпримеров к некоторым проблемам бернсайдовского типа был посвящен и доклад Е.С. Голода на Международном конгрессе математиков в Москве в 1966 г. [6].

Решение проблемы Бернсайда в случае ограниченного показателя было получено С. И. Адяном и П. С. Новиковым. Ими показано существование для любого достаточно большого нечетного натурального числа $n$ бесконечной 2-порожденной группы экспоненты $n$. Случай достаточно большого четного показателя $n$ был разобран С. В. Ивановым и И.Г. Лысёнком. Ограниченность порядков конечных групп экспоненты $n$ с фиксированным числом порождающих была доказана А. И. Кострикиным для простого $n$ и затем Е. И. Зельмановым для произвольного $n$. А. Любоцкий обнаружил присутствие неравенства Голода-Шафаревича в топологии гиперболических 3-многообразий, что помогло ему решить проблему Серра об арифметических решетках в $\mathrm{SL}_{2}(\mathbb{C})$. Проблема Куроша для PI-алгебр была положительно решена Я. Левицким и И. Капланским.

Основная часть работ Евгения Соломоновича 1970-х и 1980-х годов связана с коммутативной алгеброй, особенно с одним из важных инвариантов локальных и градуированных колец - гомологиями комплекса Козюля. В их числе характеризация (совместно с его учеником Л. Л. Аврамовым в работе [7]) горенштейновых локальных колец как колец, у которых алгебра гомологий комплекса Козюля максимального 
идеала фробениусова (одно из обобщений этой теоремы получено впоследствии Евгением Соломоновичем в работе [14]), и вычисление козюлевых гомологий и рядов Пуанкаре почти полных пересечений размерности вложения три [8].

K 1984 г. относится одна из наиболее цитируемых (наряду со статьями по теореме Голода-Шафаревича и проблемам бернсайдовского типа) работ Евгения Соломоновича [9]. Она содержит ряд результатов об относительной горенштейновой размерности: это далекое обобщение проективной размерности модулей над локальным кольцом введено Х.-Б. Фоксби. В [9], в частности, получена формула, связывающая относительную горенштейнову размерность модуля над кольцом и над факторкольцом. С горенштейновой размерностью связана и одна из последних опубликованных работ Евгения Соломоновича [17], в которой, в частности, классифицированы модули нулевой горенштейновой размерности над алгебрами Стенли-Рейснера. В работе [10] установлен ряд изоморфизмов между козюлевыми и локальными когомологиями.

Вместе с тем в 1980-е и 1990-е годы Евгений Соломонович возвращается к исследованию цепного комплекса, который появился в первом доказательстве теоремы Голода-Шафаревича: Евгений Соломонович называл его комплексом Шафаревича, хотя сейчас используется также название "комплекс Голода-Шафаревича". Это некоммутативный аналог комплекса Козюля, он получается из (дифференциально градуированной) ассоциативной алгебры присоединением новых свободных переменных, дифференциал на которых принимает значения в исходной алгебре. Евгений Соломонович исследовал как алгебры гомологий комплекса Шафаревича свободной алгебры [12], так и поведение этого комплекса и его коммутативного аналога в более общей ситуации, которая возникает при построении дифференциально градуированной модели (т. е. кофибрантной резольвенты) ассоциативной алгебры и соответственно резольвенты Тейта в коммутативном случае. В частности, им получены новые гомологические описания некоммутативных полных пересечений в смысле Аника алгебр гомологической размерности два [13]. Евгений Соломонович предложил замечательную гомологическую интерпретацию одного из главных инструментов компьютерной алгебры - коммутативных и некоммутативных базисов Гребнера и их обобщений: оказалось, что первые гомологии комплексов Козюля и Шафаревича содержат информацию о процессе построения базисов по стандартному алгоритму критических пар [11].

Работы Е.С. Голода 2000-х годов также связаны с теоретическими основаниями теории стандартных базисов в их наиболее общем виде - для фильтрованных модулей над кольцами. Эти работы установили интересные связи "бриллиантовой леммы" и дистрибутивных решеток идеалов. Среди наиболее красивых результатов - характеризация коммутативных арифметических колец как колец, над которыми справедлив критерий Бухбергера для базисов Гребнера модулей, а также теорема о том, что расширение коммутативного кольца сохраняет все базисы Гребнера в точности в том случае, когда это расширение плоское. Другие его результаты последних лет, кроме упомянутой выше работы о модулях нулевой горенштейновой размерности, также связаны с теорией колец: им получены формулы для аннуляторов модулей над коммутативными арифметическими кольцами [15] и (совместно с А. А. Туганбаевым) над инвариантными справа кольцами [16].

Для Е. С. Голода были характерны удивительная скромность, деликатность и требовательность к себе. K своим ученикам Евгений Соломонович всегда обращался с искренними интересом и доброжелательностью, щедро делясь своими идеями и участвуя в написании их первых статей. Среди учеников Е. С. Голода четыре доктора физико-математических наук - Л.Л. Аврамов, В. М. Галкин, Л. В. Кузьмин, Д. И. Пионтковский. 
В подготовке некролога активное участие приняли И. В. Аржанцев, М. В. Зайцев, А.А. Клячко, В. Т. Марков, Ю.Г. Прохоров, Д. А. Тимашев, А.А. Туганбаев, И. А. Чубаров.

В.А. Артамонов, В.М. Бухштабер, Э.Б. Винберг, Л.В. Кузъмин, В.С. Куликов, В.Н. Латышев, А.В. Михалев, А.Ю. Ольшанский, Д. О. Орлов, А.Н. Паршин, Д. И. Пионтковский

\section{Список цитированных работ Е. С. Голода}

[1] “Об одном вопросе гомологической алгебры”, УМН, 14:4(88) (1959), 157.

[2] “О кольце когомологий конечной р-группы”, Докл. АН CCCP, 125:4 (1959), 703-706.

[3] "О гомологиях некоторых локальных колец", Докл. АН СCCP, 144:3 (1962), 479-482; англ. пер.: "On the homology of some local rings", Soviet Math. Dokl., 3 (1962), 745-749.

[4] "О башне полей классов", Изв. АН СССР. Сер. матем., 28:2 (1964), 261-272 (совм. с И.Р. Шафаревичем); англ. пер.: "On class field towers", Amer. Math. Soc. Transl. Ser. 2, 48, Amer. Math. Soc., Providence, RI, 1965, 91-102 (with I. R. Šafarevič).

[5] "О ниль-алгебрах и финитно-аппроксимируемых $p$-группах", Изв. АН CCCP. Сер. матем., 28:2 (1964), 273-276; англ. пер.: "On nil-algebras and finitely approximable p-groups", Amer. Math. Soc. Transl. Ser. 2, 48, Amer. Math. Soc., Providence, RI, 1965, 103-106.

[6] "О некоторых проблемах бернсайдовского типа", Труды Международного конгресса математиков (Москва, 1966), Мир, М., 1968, 284-289; англ. пер.: "On some problems of Burnside type", Amer. Math. Soc. Transl. Ser. 2, 84, Amer. Math. Soc., Providence, RI, 1969, 83-88.

[7] "Об алгебре гомологий комплекса Козюля локального кольца Горенштейна", Матем. заметки, 9:1 (1971), 53-58 (совм. с Л.Л. Аврамовым); англ. пер.: "Homology algebra of the Koszul complex of a local Gorenstein ring", Math. Notes, 9:1 (1971), 30-32 (with L. L. Avramov).

[8] "О гомологиях некоторых локальных колец", УМН, 33:5(203) (1978), 177-178; англ. пер.: "On the homology of certain local rings", Russian Math. Surveys, 33:5 (1978), 185-186.

[9] "G-размерность и обобщенные совершенные идеалы", Алгебраическая геометрия и ее приложения, Сборник статей, Тр. МИАН СССР, 165, 1984, 62-66; англ. пер.: "G-dimension and generalized perfect ideals", Proc. Steklov Inst. Math., 165 (1985), 67-71.

[10] "Обобщенные модули частных, комплекс Козюля и локальные когомологии", Вестн. Моск. ун-та. Сер. 1. Матем., мех., 1986, № 6, 7-13; англ. пер.: "Modules of generalized fractions, Koszul complex, and local cohomologies", Moscow Univ. Math. Bull., 41:6 (1986), 6-13.

[11] "Standard bases and homology", Algebra - some current trends (Varna, 1986), Lecture Notes in Math., 1352, Springer, Berlin, 1988, 88-95.

[12] "Об алгебре гомологий комплекса Шафаревича свободной алгебры”, Фундамент. и прикл. матем., 5:1 (1999), 97-100.

[13] "Гомологии комплекса Шафаревича и некоммутативные полные пересечения", Фундамент. и прикл. матем., 5:1 (1999), 85-95. 
[14] "О двойственности в алгебре гомологий комплекса Козюля", Фундамент. и прикл. матем., 9:1 (2003), 77-81; англ. пер.: "On duality in the homology algebra of a Koszul complex", J. Math. Sci. (N. Y.), 128:6 (2005), 3381-3383.

[15] "Замечание о коммутативных арифметических кольцах", Фундамент. и прикл. матем., 19:2 (2014), 21-23; англ. пер.: "A remark on commutative arithmetic rings", J. Math. Sci. (N. Y.), 213:2 (2016), 143-144.

[16] "Аннуляторы и конечно порожденные модули", Фундамент. и прикл. матем., 21:1 (2016), 79-82 (совм. с А. А. Туганбаевым); англ. пер.: "Annihilators and finitely generated modules", J. Math. Sci. (N. Y.), 233:5 (2018), 656-658 (with A. A. Tuganbaev).

[17] "Модули нулевой горенштейновой размерности над алгебрами графов", Maтем. сб., 207:7 (2016), 81-100 (совм. с Г. А. Погудиным); англ. пер.: "Modules of Gorenstein dimension zero over graph algebras", Sb. Math., 207:7 (2016), 964-982 (with G. A. Pogudin). 